\title{
Elimination of drive exit line: transcutaneous energy transmission
}

\author{
Yuriy Pya ${ }^{1,2}$, Aigerim Abdiorazova ${ }^{3}$ \\ ${ }^{1}$ National Research Cardiac Surgery Center, Nur-Sultan, Kazakhstan; ${ }^{2}$ University Medical Center, Nur-Sultan, Kazakhstan; ${ }^{3}$ Patient Relations \\ Department, National Research Cardiac Surgery Center, Nur-Sultan, Kazakhstan \\ Correspondence to: Aigerim Abdiorazova. Patient Relations Department, National Research Cardiac Surgery Center, 38 Turan ave., 010000 , Nur- \\ Sultan, Kazakhstan. Email: abdiorazova@gmail.com.
}

Submitted Sep 15, 2020. Accepted for publication Nov 03, 2020.

doi: 10.21037/acs-2020-cfmcs-200

View this article at: http://dx.doi.org/10.21037/acs-2020-cfmcs-200

"The day will soon come when I will show the world that the transmission of power without the aid of any wires is possible."Nikola Tesla, 1885.

In the past decades development of mechanical circulatory support (MCS) devices has revolutionized the treatment of patients with advanced heart failure both as bridge-to-transplant (BTT) and destination therapy (DT). Implantation of a magnetically levitated centrifugal continuous-flow left ventricular assist device (LVAD) was found to improve results, and is associated with two-year outcomes comparable to heart transplantation (1).

After the success of the first LVADs in prolonging patient lives, attention has shifted beyond mere survival to survival with freedom from adverse events. To date, rates of adverse events post-implantation remain high, the most frequent complication being infection of the driveline (2). At the National Research Cardiac Surgery Center alone, out of 362 patients implanted with an LVAD between November 2011 and October 2020, 113 patients (31.2\%) had a driveline infection (DLI), and 78 (58.6\%) of them unfortunately died. Infection following LVAD implantation is a leading cause of morbidity, mortality, hospital readmission, and high cost of care (2).

Although many attempts have been made to tackle the issue of DLI, from antibiotic therapy (3), to development of innovative devices (4), the required percutaneous driveline poses a continuous risk of infection for the duration of support.

In Kazakhstan this problem is exacerbated by long distances between cities and regions (5). Our center is the sole clinic in the country for MCS implantation and we have trained regional coordinators on joint management of LVAD patients (5). However, it remains difficult to continuously monitor patients when they are 500 to 1,900 kilometers away from the center. This means our physicians are often unaware of a developing DLI, or discover the problem when it is already severe.

Our experience also showed that DLIs develop by the ninth month of MCS. LVAD is implanted in our center as BTT in $67 \%$ of the cases, with a hope that these patients will receive a heart transplant within 12 months. The remaining $33 \%$ of the implants are performed as DT. Those in the DT group would ideally need the transcutaneous energy transfer (TET) system in order to free the patients from possible DLI. However, organ donation is a major issue in the country (5), with an average waiting time of more than two years. As a result, only around $10 \%$ of the patients receive heart transplants. Consequently, a larger number of patients (approximately $85 \%$ of those implanted with LVAD) are considered to remain in the DT group, increasing their chances of developing DLI by the time they are on MCS support.

The ultimate solution to minimize DLI lies in total implantation of all components and energy transfer with no percutaneous drivelines. Elimination of the percutaneous driveline by integration of TET has been performed successfully in earlier designs of the LVAD and total artificial heart (TAH) (6), but is not currently incorporated into the widely used MCS systems today.

One of the first devices that used the TET system was the AbioCor TAH (6). Fourteen patients received the device as part of a clinical trial in five United States hospitals, with the longest support period at 17 months. There were no reports of serious TET system-related complications.

The AbioCor experience demonstrates that the TET system can be used safely with MCS devices, although 
careful consideration should be made regarding device type. TAH as a system is fairly complicated, which is probably why only a few widely used TAHs are available to date. Pairing TET with such complicated systems can create significant challenges. Additionally, one of the common adverse events following TAH implantation includes device failure, which generally leads to a terminal outcome, leaving little to no chance for saving the patient's life. A safer option for pairing with TET could be a choice of LVAD.

An example of this is the Arrow LionHeart LVD 2000 LVAD—-the first fully implantable system designed for DT. Six patients received the device as part of an initial trial, with a mean duration of support of 245 days. The survival rate at 18 months was $50 \%$, and no major device-related problems or pump stops were reported. One of the main advantages of this device is its design as a modular assembly. In the event of a component failure or internal battery end of life, it would allow for replacement of individual components, without need for complete replacement of the system. However, the limitations include the sizing of the device, which limits the patient eligibility criteria, and the the patient can only be uncoupled from the external power supply for up to 20 minutes.

The latest development in the field of TET incorporated with an MCS device is the fully implanted ventricular assist system (FIVAS) - a hybrid of coplanar energy transfer (CET) system (Leviticus Cardio, Ltd., Petach Tikva, Israel) with Jarvik Heart LVAD (Jarvik. Heart, Inc., New York, USA) (7).

The initial FIVAS implantation was performed in two patients at our center (7). In the early post-operative phase, CET system operation was accomplished as expected in both patients. The CET system has an externalized retroauricular bailout connector, which provided a pragmatic solution to balance safety and efficacy validation in our early investigational experience.

The highest achievement of this system is that it gives the patient the ability to swim and exercise-activities that any patient on regular MCS only would only dream to do (8).

The novel CET system is similar to the original TET design, but is unique by incorporating two large rings with coil-within-the-coil topology to ensure strong resonance power (7). The battery is charged wirelessly with a belt worn around the chest, and a user watch notifies the patient when the internal battery needs charging. The internal battery can provide greater than eight hours of continuous untethered operation (7). These features of the CET system are crucial, since a true goal of TET is to give the patients total freedom from continuous or frequent charging, or having to wear a heavy support system, rather than merely prolonging their lives and freeing them from adverse events.

Further validation of the CET system is needed by longer-term safety and efficacy demonstration in larger patient numbers. However, our initial experience shows that not only is it possible to have an LVAD without percutaneous access drive, but is absolutely necessary to achieve the next level quality of life for our patients.

\section{Acknowledgments}

Funding: None.

\section{Footnote}

Conflicts of Interest: The authors have no conflicts of interest to declare.

Open Access Statement: This is an Open Access article distributed in accordance with the Creative Commons Attribution-NonCommercial-NoDerivs 4.0 International License (CC BY-NC-ND 4.0), which permits the noncommercial replication and distribution of the article with the strict proviso that no changes or edits are made and the original work is properly cited (including links to both the formal publication through the relevant DOI and the license). See: https://creativecommons.org/licenses/by-nc-nd/4.0/.

\section{References}

1. Mehra MR, Goldstein DJ, Uriel N, et al. Two-Year Outcomes with a Magnetically Levitated Cardiac Pump in Heart Failure. N Engl J Med 2018;378:1386-95.

2. Gustafsson F, Netuka I. Interplay of pump design elements and bleeding predilection-Mechanisms for a forward momentum. J Heart Lung Transplant 2019;38:817-9.

3. Leuck AM. Left ventricular assist device driveline infections: recent advances and future goals. J Thorac Dis 2015;7:2151-7.

4. Kantrowitz, AB. Percutaneous Access Device System Facilitating Cell Growth Thereon. 2010. U.S. Patent $7.704,225$.

5. Pya $\mathrm{Y}$, Bekbossynova $\mathrm{M}$, Jetybayeva $\mathrm{S}$, et al. Initial 3-year outcomes with left ventricular assist devices in a country with a nascent heart transplantation program. ESC Heart Fail 2016;3:26-34.

6. Smith PA, Cohn WE, Frazier OH. Chapter 7-Total 
Artificial Hearts. In: Gregory SD, Stevens MC, Fraser JF. editors. Mechanical Circulatory and Respiratory Support. Elsevier ,2017.

7. Pya Y, Maly J, Bekbossynova M, et al. First human use of a wireless coplanar energy transfer coupled with a continuous-flow left ventricular assist device. J Heart Lung
Transplant 2019;38:339-43.

8. Gizitdinov N. Heart Pump With Wireless Charging Implanted by Kazakh Doctors. 2019. Available online: https://www.bloomberg.com/news/articles/2019-02-06/ wireless-device-for-heart-pumps-implanted-inkazakhstan-center

Cite this article as: Pya Y, Abdiorazova A. Elimination of drive exit line: transcutaneous energy transmission. Ann Cardiothorac Surg 2021;10(3):393-395. doi: 10.21037/acs-2020-cfmcs-200 